The Chittagong Univ. J. Sci. 41(1) : 112-121, 2019

DOI: https://doi.org/10.3329/cujs.v41i1.51919

\title{
A New Solution of Intermediate Cosmological Inflation with the Effect of Scalar Field
}

\author{
Shomi Aktar ${ }^{1}$ and Anjan Kumar Chowdhury ${ }^{2 *}$ \\ 1. Department of Mathematics, University of Chittagong, Chittagong-4331, \\ Bangladesh \\ 2. Jamal Nazrul Islam Research Centre for Mathematical and Physical Sciences, \\ University of Chittagong, Chittagong-4331, Bangladesh \\ *Corresponding Author; E-mail:anjan.kumar@cu.ac.bd
}

\begin{abstract}
According to the inflationary model, the universe had a brief period of extraordinarily rapid expansion or inflation during which its diameter increased by a factor at least $10^{25}$ times larger than previously thought. In this work an analysis is given on inflationary universe, which expands at a rate intermediate between that of power-law and exponential inflation. We have examined the model of Barrow which is solved exactly and leaded to power law inflation. We have tested a new potential by applying the scalar field using the equation of motion and found some new interior solutions.
\end{abstract}

Keywords: Inflation, scalar field, expansion, cosmological constant, power law.

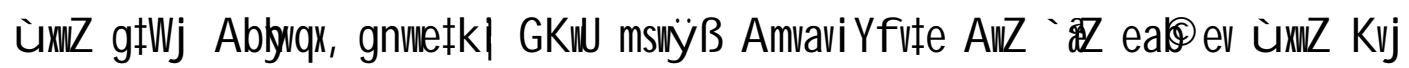

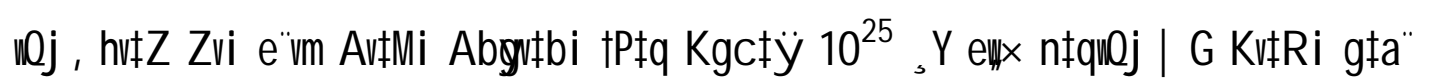

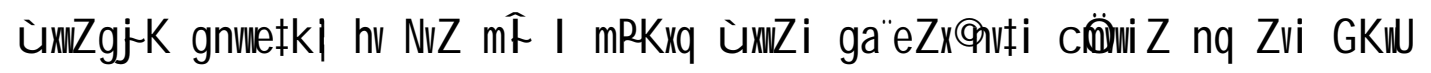

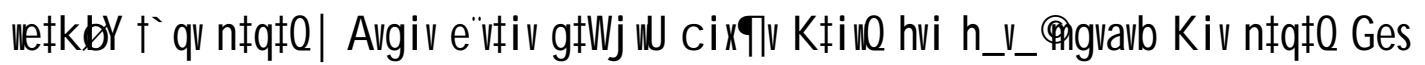




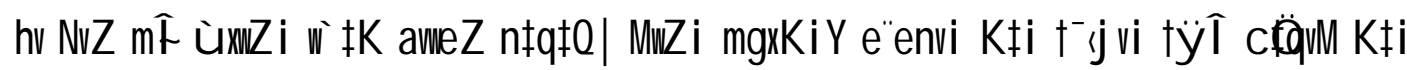

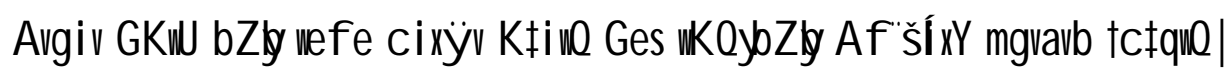

\section{Introduction}

The expansion rate of "intermediate inflation" lies between the exponential expansion and power law expansion but corresponding accelerated expansion does not start at the onset of cosmological evolution, for example, by scalar fields with purely exponential potentials or scalar-tensor gravity theories in which the BransDicke theory is extended so that the constant Brans-Dicke parameter $\omega$ becomes an increasing power-law function of the Brans-Dicke scalar field [5]. However, it is possible to parameterize all of these possibilities by a simple equation of state in which the pressure $p$ and the density $\rho$ are related by the non-linear relation [1]

$$
p+\rho=\gamma \rho^{\lambda},
$$

where $\gamma$ and $\lambda$ are constants. The two defining equations for the scale factor $R(t)$ of a Friedman universe with curvature parameter $k(8 \pi G=c=1)$ are

$$
\begin{gathered}
2 H^{2}=\rho-k R^{-2}, \\
\dot{\rho}+3 H(p+\rho)=0,
\end{gathered}
$$

where the Hubble expansion rate is defined as $H \equiv \frac{\dot{R}}{R}$.

Hence when $\lambda=1$ the equation of state (1) reduces to that of a standard perfect fluid equation of state. When $k=0$ and $\gamma=0$, the system (2),(3) has the exponential solution

$$
R(t)=\exp \left(H_{0} t\right), H_{0} \text { is a constant } .
$$

When $\gamma \neq 0$,it has the power-law inflationary solution [3-5] 
114 A New Solution of Intermediate Cosmological Inflation with the Effect of Scalar Field

$$
R(t)=t^{2 / 3} \gamma,
$$

when $2 / 3>\gamma>0$. Both (4) and (5) are generated by particular scalar field potentials $[2,6,8]$ of the form

$$
V=V_{0} \exp (\alpha \varphi)
$$

with $3 \gamma=\alpha^{2}$.

However, when $\lambda \neq 1$ new types of inflation can arise from the stress (1). Primarily in the case $\gamma>0$ and $\lambda>1$, this generates a new form of intermediate inflation $[1,10]$ dependents on cosmic time $t$ in which

$$
R(t)=\exp \left(A t^{f}\right),
$$

where $\mathrm{A}$ is a positive constant and

$$
f=\frac{2(1-\lambda)}{1-2 \lambda} .
$$

We see that $0<f<1$ and we have slower than exponential inflation. In this case it is also possible to rewrite the model fluid stress (1) as that of a scalar field with self-interaction potential $V(\varphi)$ of the form [1]

$$
V(\varphi)=\frac{48 A^{2}}{(\beta+4)^{2}}\left(\frac{\varphi-\varphi_{0}}{(2 A \beta)^{1 / 2}}\right)^{-\beta}-\frac{4 A \beta}{(\beta+4)^{2}}\left(\frac{\varphi-\varphi_{0}}{(2 A \beta)^{1 / 2}}\right)^{-\beta-2},
$$

with

$$
\varphi=\varphi_{0}+(2 A \beta t)^{1 / 2},
$$

where $\varphi_{0}$ is a constant and

$$
\beta \equiv 4\left(f^{-1}-1\right) .
$$

Here, we shall investigate the behavior of the intermediate inflationary universes of the type (7). Although, as we have just pointed out, it is possible to derive such solutions from explicit chaotic inflationary potentials or from Brans-Dicke models, 
it is rather difficult to represent them in this manner and so we shall discuss them in terms of the parameters $p$ and $\rho$ of ( 1$)$ where, $k=0$,

$$
\rho=3 H^{2}=3 A^{2} f^{2} t^{2 f-2} .
$$

A feature of matter fields of this type is that they produce a Hubble rate that falls off more slowly than an inverse power of time. In the limit that $f \rightarrow 1$ the de Sitter behaviour is recovered and the density is a constant. We shall now investigate whether intermediate inflationary universes satisfy a no-hair theorem of the type found for exponential and power-law inflationary universes. In particular, we shall determine whether the solution of the Friedman equations for zero curvature, given by equations (1), (7) and (12), is stable against density, velocity and gravitationalwave perturbations. We shall use the formalism of Weinberg [2] and consider a linearization of the Einstein equations around the background Friedman metric $\tilde{g}_{i j}$ defined by the solutions (1), (7) and (12). We expand the metric as

$$
g_{i j}=\tilde{g}_{i j}+h_{i j}
$$

Here, $\tilde{g}_{i j}$ are the background and $h_{i j}$ are the small perturbations. The metric $h_{i j}$ can be decomposed into scalar, vector and transverse-traceless tensor modes. Consider $h_{k k}=0$ for first transverse-traceless (gravitational wave) perturbations [14]. They satisfy the propagation equation

$$
\ddot{h}_{i j}-\frac{\dot{R}}{R} \dot{h}_{i j}+\left(\frac{k^{2}}{R^{2}}-2 \frac{\ddot{R}}{R}\right) h_{i j}=0,
$$

where $k$ is the gravitational wave number and the derivatives are with respect to the commoving proper time t. For long-wavelength limit $k \rightarrow 0$ we can neglect the $k^{2} R^{-2}$ term. Using the background of intermediate inflation defined by (1), (7) and (12), the equation (14) then becomes 
116 A New Solution of Intermediate Cosmological Inflation with the Effect of Scalar Field

$$
\ddot{h}_{i j}-A f t^{f-1} h_{i j}-\left[2 A f(f-1) f^{f-2}+2 A^{2} f^{2} t^{2 f-2}\right] h_{i j}=0 \text {. }
$$

After a series of changes of variables we can write the exact solution of (15) in terms of the confluent hypergeometric functions [1-3] $\mathrm{M}$ and $\mathrm{U}$ as follows

$$
h_{i j}=t \exp \left(-A t^{f}\right)\left[B_{1} M\left(1, f+\frac{1}{f}, 3 A t^{f}\right)+B_{2} U\left(1, f+\frac{1}{f}, 3 A t^{f}\right)\right],
$$

where $B_{1}$ and $B_{2}$ are integration functions of the spatial coordinates alone. As $t \rightarrow \infty$ the solution has the asymptotic form

$$
h_{i j}=B_{1}(x) \exp \left(2 A t^{f}\right)+B_{2}(x) t^{1-f} \exp \left(-A t^{f}\right),
$$

The second mode decays until the first grows only as fast as the background metric whose spatial components grow as $R^{2}(t)=\exp \left(2 A t^{f}\right)$. Thus there are no growing metric movements and the space-time will appear increasingly homogeneous and isotropic within the event horizon of a geodesically moving observer [9]. When $f \rightarrow 1$ the known stability of the de Sitter model can be recovered. In general the metric distortion does not damp to zero. There exists a distortion evolved by the spatial functions $B_{1}(x)$ and $B_{2}(x)$ which reflects the fact that no global homogenization occurs beyond the horizon of a geodesic observer [1].

Both modes and the associated metric perturbation decay with time with respect to the Friedman background $R(t) \propto \exp \left(A t^{f}\right)$. Let us consider a spherical overdensity in a $k=0$ Friedman universe; the perturbation behaves like an isolated $k=+1$ Friedman model. If we consider the right-hand side of (2) we see that, with the solutions (7) and (12) for the $k=0$ background, the two terms there vary as $\rho \propto t^{2 f-1}$ and $k R^{2}(t) \propto \exp \left(A t^{f}\right)$ respectively, and so the behaviour of the $k=+1$ 
overdensity approaches that of the $k=0$ background asymptotically; that is, the perturbation decays.

This argument for the decay of density perturbations also establishes the fact that intermediate inflationary models will resolve the flatness problem in the context of isotropic universes. The influence of the curvature term decreases relative to that of the energy density term on the right-hand side of (2) just as it does for the exponential and power-law inflationary models [10] with $p=(\gamma-1) \rho$ and $0 \leq \gamma<\frac{2}{3}$

\section{New Solutions throughout the Barrow's Inflationary Model}

The density and pressure of the scalar field [11] are

$$
\begin{aligned}
& \rho=\frac{1}{2} \dot{\varphi}^{2}+V(\varphi), \\
& p=\frac{1}{2} \dot{\varphi}^{2}-V(\varphi),
\end{aligned}
$$

where the scalar field $\varphi$ is massless provided $\varphi=\varphi(t)$ only and $V(\varphi)$ is potential energy.

For the case where potential energy, $P E=V(\varphi)=0$, we have $p=\rho$ i.e. the pressure and the density is same. In this case the pressure is positive and cannot be interpreted as a variable or cosmological constant.

For the case where kinetic energy, $K E=\frac{1}{2} \dot{\varphi}^{2}=0$, we have $p=-\rho$, which means that $\rho$ can be interpreted as a strong cosmological constant. 
118 A New Solution of Intermediate Cosmological Inflation with the Effect of Scalar Field

Interpreting $\rho$ as a cosmological constant leads us to expect that a weak cosmological constant can vary [12]. This follows from $\rho=$ constant or $\rho=\rho(R)$ giving a variable function (but a strong cosmological constant cannot vary).

These results are true in general when $V(\varphi)$ isn't specified yet.

Let us now consider a new potential for Barrow model

$$
V(\varphi)=\beta e^{-\lambda \varphi},
$$

we find that $\dot{\varphi}(t)=\frac{2}{\lambda} \ln t$ and $\beta=\frac{2}{\lambda^{2}}\left(\frac{6}{\lambda^{2}}-1\right)$ for $8 \pi G=1$.

As $\mathrm{A}$ is an arbitrary constant, we $\operatorname{consider} A \equiv \frac{\lambda}{2}$ for a suitable solution. From equations (9), (10), (11) we get new value of $\beta, \varphi(t)$ and $V(\varphi)$ as

and

$$
\begin{aligned}
& \beta=A(3 A-1), \\
& \varphi(t)=\sqrt{2 A} \ln t, \\
& V(\varphi)=A(3 A-1) e^{\frac{2}{A}} .
\end{aligned}
$$

Now the density becomes,

and

$$
\begin{gathered}
\rho=\frac{3 A^{2}}{t^{2}}, \\
\rho_{\text {Barrow }}=\frac{3 A^{2}}{t^{2}}\left(\frac{2 A}{3}-1\right)=\left(\frac{2 A}{3}-1\right) \rho .
\end{gathered}
$$

The general equation of state is $p=\frac{\gamma}{3} \rho$, gives the Barrow equation of state

$$
\gamma_{\text {Barrow }}=\frac{2}{\lambda}-3 \text {. }
$$

But the power law inflation results for $A>1$. Substituting this into the equation (27) implies

$$
\gamma_{\text {Barrow }}>-3 \text {, }
$$


which we expect because power law inflation implies $\ddot{R}>0$. Thus for $A>1$ the Barrow pressure is negative with $\gamma<-1$ and thus $\rho=\frac{3 A^{2}}{t^{2}}$ corresponds to a weak cosmological constant. Furthermore, this cosmological constant is variable and decays with time.

Again if we put another type of potential

$$
V(\varphi)=V_{0} e^{A \varphi} \text { and }(t)=(2 A t)^{1 / 2},
$$

it gives

$$
\begin{gathered}
V^{\prime}(\varphi)=A V_{0} e^{A \varphi}=A V(\varphi), \\
\dot{\varphi}(t)=\sqrt{\frac{A}{2}} t^{-\frac{1}{2}}, \\
\ddot{\varphi}(t)=-\sqrt{A}\left(\frac{1}{2 t}\right)^{\frac{3}{2}} .
\end{gathered}
$$

Then we get

$$
\ddot{\varphi}+3 H \dot{\varphi}+V^{\prime}(\varphi)=0,
$$

which is the equation of motion [11]. It follows the expansion rate as

$$
\frac{\dot{R}}{R}=\frac{1}{3}\left[\frac{1}{2 t}-\sqrt{2 t A} V(\varphi)\right],
$$

and we get a new solution as

$$
R(t)=c t^{\frac{1}{6}}-\exp \left[\frac{V(\varphi) \sqrt{A}}{3}(2 t)^{\frac{3}{2}}\right] .
$$

This expansion includes Power law inflation as well as exponential expansion [7].

\section{Conclusions}

The general concept of inflation rests on being able to achieve a negative pressure equation of state. In the case of inflationary model, we have performed first steps in 
120 A New Solution of Intermediate Cosmological Inflation with the Effect of Scalar Field

the study of the behavior of the intermediate inflation and we have found solutions for which one can obtain the two inflationary epochs and one matter dominated epoch. Present study of intermediate inflation reveals that it admits scaling solution and has got a natural exit form it at a later epoch of cosmic evolution, leading to late time acceleration. In this work we have calculated a type of expansion in which both the power law and exponential expansion [3, 4] like de-Sitter model. The Power law inflation is directly involved time-time which refers in the first term and the second term represents the exponential (de-Sitter) expansion [13]. For now, inflation is the extension of the standard cosmological model that looks most likely to survive. The power law inflationary universe model induced by a scalar field with an exponential potential is studied. The result shows that the degree of inflation is small for small initial values of the scalar field $\varphi$, and it grows exponentially with increasing $\varphi_{0}(\varphi$ at $t=o)$. This means that most of the physical volume of the universe comes into being not by virtue of the expansion of regions which initially and randomly contained a small field $\varphi$.

\section{References}

[1] J. D. Barrow and Paul Saich: The Behaviour of Intermediate Inflationary Universes, Phys. Lett. B,1990, 235 , 40.

[2] S. W. Weinberg: "Gravitation and Cosmology: Principles and Applications of the General Theory of Relativity", Wiley \& Sons, New York, 1972, p. 244.

[3] W. Boucher and G. Gibbons: "The Very Early Universe”, (eds). G. Gibbons, S. W. Hawking and S.T.C. Siklos, Cambridge University Press, Cambridge, 1987, 943. 
[4] L. Jensen and J. Stein-Schabes: Phys. Rev. D,1987, 35,1146.

[5] A. B. Burd and J. D. Barrow: Nucl. Phys. B 1988, 308, 929.

[6] A. D. Linde: Lett. Nuovo Cim., 1984, 39, 401.

[7] L. F. Abbott and M. B. Wise: Nucl. Phys. B, 1984, 244, 541.

[8] A. H. Guth, The Inflationary Universe, A Possible Solution to The Horizon and Flatness Problems, Phys. Rev. D, 1981, 23, 347.

[9] M. A. H. Mac Callum: "Cargese Lectures in Physics" Vol. 6,Gorden and Breach, New York, 1973, p. 61.

[10] R. Herrera and N. Videla1: Eur. Phys. J. C, 2018, 298, 933.

[11] R. Herrera, M. Olivares and N. Videla: Eur. Phys. J. C, 2013, 73, 2475.

[12] S. Aktar: "Studies on FRW Model and Inflationary Model of the Universe", M.S. Thesis, Department of Mathematics, University of Chittagong, 2010.

[13] J. N. Islam: "An Introduction to Mathematical Cosmology", First edition, Cambridge University Press, UK, 1983, p. 128.

[14] M. H. A. Biswas: "Mathematics and Physics of Collapsing Stars", First edition, VDM Verlag, Germany, 2010, p. 146.

Manuscript received on 19 March, 2019, Revised manuscript received on 03 September, 2019 and accepted on 04 September, 2019

The Chittagong Univ. J. Sc. Vol. 41(1), 2019 